\title{
ELECTRON-ION RECOMBINATION AT SUB-ZERO TEMPERATURE
}

\author{
S. Bhattacharyya AND A. Roy \\ Gokhale Memorial Girls' College \\ Calcutta - 700 020, India
}

(Received November 6, 1998)

\begin{abstract}
In this paper we have considered two-step radiative recombination channel over the existing spontaneous radiative recombination channel to explain the causes of experimentally obtained enhanced recombination rate at sub-zero temperature. The existing recombination rate due to spontaneous radiative recombination is 4 times smaller than the recent experimental data. In the case of $\mathrm{Ne}^{10+}$ we obtain good agreement between theory and experiment when contribution from two-step radiative recombination channel is added to the spontaneous radiative recombination channel. Recombination rate for $\mathrm{Ar}^{18+}$ is calculated. The radiation spectrum given out by two-step radiative recombination and spontaneous radiative recombination in the recombination rate processes are discrete spectrum and continuous spectrum, respectively. Experimental identification of these two channels through the spectral signatures is suggested.
\end{abstract}

PACS numbers: $32.70 . \mathrm{Fw}, 34.80 . \mathrm{Kw}$

\section{Introduction}

Among the fundamental electron-ion interactions which occur in plasma [1, 2] recombination at low energies still intrigues the physicists. Measurements performed under most precise conditions yet achieved have constantly yielded experimental results 4 to 50 times above the existing theoretical expectations. No convincing explanation of the enhanced rates exists. New experimental accesses to the study of the radiative recombination $(R R)$ have become available through the use of heavy ion storage rings with electron coolers. The result of the high resolution measurements of the $\mathrm{RR}$ rates for highly charged ions in the sub $\mathrm{meV}$ region clearly exhibits the energy dependence of the deviation between existing $\mathrm{RR}$ theory and measured results.

*Mailing address: 370/1, N.S.C. Bose Road, Calcutta-700047, India. 
Radiative recombination is a significant ion loss mechanism during electron cooling of ions in a storage ring, where zero average relative electron-ion velocity is enforced, since its rate coefficient peaks at low electron-ion velocity. Thus the detection of recombination is often used as a diagnostic tool for electron cooling, as it reveals information about the CM energy distribution of electrons in the cooler ring. The study of $R R$ is also important to understand fundamental processes in reactions of free electrons with ions, and diagnostics in astrophysical and fusion plasmas.

Radiative recombination is defined as the capture of free electron $e^{-}$by an ion of charge $q$ accompanied by a photon $\gamma$ :

$$
\mathrm{A}^{q+}+\mathrm{e}^{-} \rightarrow \mathrm{A}^{(q-1)+}(n)+\gamma
$$

where $n$ is the principal quantum number. The prototype RR process (1) with completely stripped projectiles must involve three particles since simultaneous conservation of energy and momentum strictly precludes the formation of hydrogen like atomic system without the presence of an external particle or field. This additional particle is the photon, which carries off the excess energy in the case of RR.

The pioneering theoretical study of RR was carried out by Kramers [3] within a semi-classical approach. The quantum mechanical first order perturbation theory for the same problem was originally devised by Gordon [4] and subsequently implemented by Stobbe [5]. Because of its simplicity, Kramers' formula is still frequently used to calculate the RR cross section by applying a correcting Gaunt factor, defined as a quantum mechanical Stobbe and semi-classical Kramers cross sections. The initial recombination studies in merged beam single pass experiments showed good agreement between RR theory by Kramers and experimental results. However, in the storage ring experiments, deviations from theoretical $R R$ predictions by Kramers have been found for a. highly charged ion. Below $10 \mathrm{meV}$ the measured rate coefficient for $\mathrm{Ne}^{10+}$ is found higher by a factor of 4 as compared to the Kramers calculation [6].

In the present paper we have tried to view the phenomena of $R R$ as due to a combined effect of two distinct processes: spontaneous radiative recombination (SRR) and two-step radiative recombination (TSRR). In the TSRR channel, first theorized in Ref. [7] by one of the authors (SB), an emitted photon is rather discrete. In the TSRR channel recombination occurs in an intermediate excited state which to conserve momentum emits a discrete photon and goes to a lower state. In the SRR channel recombination may occur in any of the states and the photon carries away the extra energy giving rise to a continuous spectrum. Sums of the recombination rates from the two channels compare well with the experimental enhanced results. The dominant interaction mechanism in the SRR channel is a dipole interaction. The TSRR is a second order process where both the Coulomb and the dipole interactions take part. We use field theory and Coulomb gauge to calculate these processes. In the present paper we have calculated the $R R$ rate coefficients of bare $\mathrm{Ne}$ and $\mathrm{Ar}$ ions and compared the same with the existing experimental rate coefficients of bare $\mathrm{Ne}$ ion for the $\mathrm{CM}$ energy range $0.01 \mathrm{meV}$ to $0.1 \mathrm{eV}$. For energy range up to $1 \mathrm{meV}$ the $\mathrm{RR}$ rate from the SRR channel is greater than those from the TSRR channel (Figs. 1a, b). Above this 
energy the SRR rate drops rapidly while the TSRR rate decreases slowly but is always remaining greater than the SRR rate. Below $1 \mathrm{meV}$ the sum of the SRR and the TSRR rates are closer to the experimental results [8] but greater than the calculated rate coefficients based on the Kramers cross section. Above $1 \mathrm{meV}$ the present calculation and Kramers results follow the experimental curve.

\section{Theoretical formalism}

The radiative recombination reactions under consideration for SRR and TSRR processes are, respectively:

$$
\mathrm{A}^{z+}+\mathrm{e}^{-} \rightarrow \mathrm{A}^{(z-1)+}(1 s)+\gamma
$$

and

$$
\mathrm{A}^{z+}+\mathrm{e}^{=} \rightarrow \mathrm{A}^{(z-1)+}(n, l) \rightarrow \mathrm{A}^{(z-1)+}(1 s)+h \nu .
$$

To apply field theory in calculating the $R R$ rate we assumed the bare nucleus as an elementary particle and corresponding annihilation operator being taken as $B_{1}$. We have already argued in our previous paper [9] that "the elementariness of a particle is an energy dependent phenomenon". Hadron that behaves as an elementary particle in quantum electrodynamics (QED) begins to show a structure with quark flavour in the quantum chromodynamics (QCD) domain. Eventually, a bare nucleus, when subjected to energy far below its binding energy, will obey Fermi statistics or Bose statistics depending on their resultant spin. $\mathrm{Ne}^{10+}$ and $\mathrm{Ar}^{18+}$ will behave like Bose particles with integral spins. $a_{q}, B_{1}$ and $C_{k}$ are respectively the annihilation operators for electron, nucleus, and photon with sufficed momenta. The state vectors of the interacting systems for the initial state is

$$
\left|\Psi_{\mathrm{i}}\right\rangle=\exp \left(E_{\mathrm{i}} t / \hbar\right) \psi_{\mathrm{c}}(r) a_{q 1}^{\dagger} B_{11}^{\dagger}|0\rangle
$$

The intermediate state is

$$
\left|\Psi_{\mathrm{I}}\right\rangle=\exp \left(E_{\mathrm{I}} t / \hbar\right) \varphi_{n l}^{\prime}(r) a_{q 2}^{\dagger} B_{12}^{\dagger}|0\rangle
$$

The final state vector containing an atom and a photon is given by

$$
\left|\Psi_{\mathrm{f}}\right\rangle=\exp \left(E_{\mathrm{f}} t / \hbar\right) \varphi_{n !}^{z-1}(r) a_{q 2}^{\dagger} B_{12}^{\dagger} C_{k}^{\dagger}|0\rangle
$$

$\psi_{\mathrm{c}}(r)$, the Coulomb distorted plane wave for the incident electron in the field of nucleus is given by

$$
\psi_{\mathrm{c}}(r)=F_{\mathrm{c}}(p) \exp (\mathrm{i} p r), \quad \text { where } \quad F_{\mathrm{c}}(p)=\sqrt{\frac{2 \pi m e^{2} z}{\hbar^{2} p}},
$$

$p$ is the relative momentum of the electron with respect to the target. $\varphi_{n l}^{z-1}(r)$ is the bound state of the one-electron ion. The $S$-matrix of the two-body radiative recombination is

$$
S=1+H_{1}+H_{2}+H_{1} H_{1}+H_{1} H_{2}+H_{2} H_{2}+\text { higher order terms, }
$$

$H_{1}$ is the interaction Hamiltonian between electron and electromagnetic field, $H_{2}$ is the Coulomb interaction between electron and the bare ion

$$
H_{1}=\frac{e}{m c} p \boldsymbol{A}+\frac{e^{2}}{2 m c^{2}} \boldsymbol{A}^{2}
$$


$\boldsymbol{p}$ is the momentum operator and $\boldsymbol{A}$ is the vector potential

$$
\boldsymbol{A}=\sum_{k} \sqrt{\frac{2 \pi \hbar c^{2}}{\omega_{k}}} u_{k}\left[c_{k} \exp (-\mathrm{i} k x)+c_{k}^{\dagger} \exp (\mathrm{i} k x)\right],
$$

$u_{k}$ is the polarization vector and $k$ is the photon momentum. For a single photon emission the first term of $H_{1}$ is considered. The Coulomb interaction $H_{2}$ is given by

$$
H_{2}=\int \frac{\rho(x) \sigma\left(x^{\prime}\right)}{\left|x-x^{\prime}\right|} \mathrm{d}^{3} x \mathrm{~d}^{3} x^{\prime},
$$

$\rho(x)$ and $\sigma(x)$ are the charge densities for electron and nucleus, respectively,

$$
\begin{aligned}
& \rho(x)=-e \varphi(x) \varphi^{*}(x), \\
& \sigma(x)=z e \Theta(x) \Theta^{*}(x),
\end{aligned}
$$

$\varphi(x)$ and $\Theta(x)$ are the field operators for electron and nucleus, respectively,

$$
\begin{aligned}
& \varphi(x)=\sum_{f} \int \kappa_{f} a_{s}^{\dagger} \exp (\mathrm{i} s x) \mathrm{d}^{3} s, \\
& \Theta(x)=\int B_{s^{\prime}}^{\dagger} \exp \left(\mathrm{i} s^{\prime} x\right) \mathrm{d}^{3} s^{\prime},
\end{aligned}
$$

$\kappa_{\mathrm{f}}$ is the Pauli spinor for electron. The amplitude $M_{\mathrm{s}}$ for spontaneous radiative recombination term is given by

$$
M_{\mathrm{s}}=\left\langle\psi_{\mathrm{f}}\left|H_{1}\right| \psi_{\mathrm{i}}\right\rangle
$$

The second order process TSRR has the amplitude $M_{\mathrm{T}}$ given by

$$
M_{\mathrm{T}}=\frac{\left\langle\Psi_{\mathrm{f}}\left|H_{1}\right| \Psi_{\mathrm{I}}\right\rangle\left\langle\Psi_{\mathrm{I}}\left|H_{2}\right| \Psi_{\mathrm{i}}\right\rangle}{E_{\mathrm{I}}-E_{\mathrm{f}}+\mathrm{i} \eta}
$$

Calculation for SRR and TSRR cross sections is similar to those in the paper by Bhattacharyya et al. [10].

\section{1. $S R R$ cross section}

The amplitude for SRR after some calculation is obtained as

$$
M_{\mathrm{s}}=\bar{C}\left(\frac{-\mathrm{im} \pi}{4 \hbar}\right)(\hbar \omega) \int \varphi_{n I}(r) \psi_{\mathrm{c}}(r) r \sin 2 \theta \mathrm{d}^{3} r,
$$

where $\bar{C}=(e / m c) \sqrt{2 \pi \hbar c / \omega}$ and $\theta$ is the angle between $p$ and $k$. The quantities $p^{\prime}$ and $E^{\prime}$ are the momentum and energy of the one-electron ion and $\varepsilon_{n l}$ is the corresponding binding energy. From the energy conservation $E^{\prime}=U-\hbar \omega$, where $U=E_{\mathrm{i}}+\varepsilon_{n l}$.

For the capture into $n l$ states the SRR cross section becomes

$$
\sigma_{n l}^{\mathrm{s}}\left(E_{\mathrm{i}}\right)=\frac{m}{|p|} \frac{2 \pi}{\hbar} \int \delta\left(E_{\mathrm{i}}-E_{\mathrm{f}}\right)\left|M_{\mathrm{s}}\right|^{2} \frac{\mathrm{d}^{3} k \mathrm{~d}^{3} p^{\prime}}{(2 \pi)^{6}} .
$$

Since $\mathrm{d}^{3} k=\left(\omega^{2} / c^{3}\right) \mathrm{d} \omega \sin \theta \mathrm{d} \theta \mathrm{d} \varphi$ and $\mathrm{d}^{3} p=4 \pi m \sqrt{2 m E^{\prime}} \mathrm{d}^{3} E^{\prime}$ and $\omega$ varies from 0 . to $U / \hbar$, the cross section becomes

$$
\sigma_{n l}^{\mathrm{s}}\left(E^{\prime}\right)=\frac{z \sqrt{2}}{15 c^{3}|\bar{p}|^{2}} I_{n l}^{2} \int_{0}^{U / \hbar} \omega^{3}(U-\hbar \omega)^{1 / 2} \mathrm{~d} \omega
$$




$$
=\frac{z \sqrt{2}}{15 c^{3} h^{4}} \frac{11}{5} I_{n l}^{2} \frac{\left(E_{\mathrm{i}}+\varepsilon_{n l}\right)^{4}}{2 m E_{\mathrm{i}}},
$$

where $I_{n l}=\int \varphi_{n l}(r) r \exp (\mathrm{ipr} r) \mathrm{d}^{3} r$.

Since the relative energy $\left(E_{\mathrm{i}}\right)$ of the super cool electron under consideration is of the order of $\mathrm{meV}, \mathrm{Eq}$. (17) shows that SRR cross section is inversely proportional to $E_{\mathrm{j}}$. Cross section also depends on the overlap integral $I_{n l}$.

\subsection{TSRR cross section}

From (16) the TSRR amplitude

$$
\begin{aligned}
& M_{\mathrm{T}}=\frac{M_{1} M_{2}}{\left|E_{\mathrm{I}}-E_{\mathrm{f}}+\mathrm{i} \eta\right|}, \\
& M_{1}=\left\langle\Psi_{\mathrm{f}}\left|H_{1}\right| \psi_{\mathrm{I}}\right\rangle, \quad M_{2}=\left\langle\Psi_{\mathrm{I}}\left|H_{2}\right| \psi_{\mathrm{i}}\right\rangle,
\end{aligned}
$$

$M_{2}$ is the amplitude for recombination into an excited intermediate state by Coulomb interaction. $M_{1}$ is the amplitude for transition to the ground state by dipole interaction with emission of discrete photon. The cross section for TSRR then becomes

$$
\sigma_{n^{\prime} l}^{\mathrm{T}}\left(E_{\mathrm{i}}\right)=\tau_{n^{\prime} l^{\prime}-n l}^{-1} \frac{m}{\hbar|p|} \int \frac{\left|M_{2}\right|^{2}}{\left(E_{\mathrm{i}}-E_{\mathrm{I}}\right)^{2}} \frac{\mathrm{d}^{3} p^{\prime}}{(2 \pi)^{3}},
$$

where the clecay rate for transition from $n l$-state to $n^{\prime} l^{\prime}$-state is

$$
\begin{aligned}
\tau_{n^{\prime \prime} \prime}^{-1} \rightarrow n l & =\frac{2 \pi}{\hbar} \int \partial\left(E_{\mathrm{i}}-E_{\mathrm{f}}\right)\left|M_{1}\right|^{2} \frac{\mathrm{d}^{3} k}{(2 \pi)^{3}} \\
= & \frac{4 e^{2}}{3 \hbar}\left(\frac{\Delta E_{n^{\prime} l^{\prime \prime} \rightarrow n l}}{\hbar c}\right)^{3}\left[4 \sqrt{2}\left(\frac{2}{3}\right)^{5} \frac{a_{0}}{z}\right]^{2} .
\end{aligned}
$$

Here $a_{0}$ is the Bohr radius, $\Delta E_{n^{\prime \prime} l^{\prime} \rightarrow n l}$ is the energy for transition $n^{\prime} l^{\prime} \rightarrow n l$.

Knowing that $E_{\mathrm{I}}=E_{\mathrm{i}}+\varepsilon_{n^{\prime} l^{\prime}}$, we get after some lengthy calculations

$$
\sigma_{n^{\prime} l^{\prime}}^{\mathrm{T}}\left(E_{\mathrm{i}}\right)=\tau_{n^{\prime} l^{\prime}-n l}^{-1} \frac{e^{4}}{\left.(2 \pi)^{3}\right)} \frac{m}{|p|} J_{n^{\prime} l^{\prime}}^{2} \frac{8 \pi m \sqrt{2}}{3} \frac{\left(E_{\mathrm{i}}+\varepsilon_{n l}\right)^{3 / 2}}{\left(\varepsilon_{n^{\prime} l^{\prime}}\right)^{2}},
$$

where

$$
J_{n^{\prime} l^{\prime}}=\int \frac{\psi_{n^{\prime} l^{\prime}}(r) \psi_{\mathrm{c}}(r)}{|r|} \mathrm{d}^{3} r
$$

\subsection{Recombination rate}

The recombination rate in the SRR channel for recombination into $n l$ state is

$$
\alpha_{n l}^{\mathrm{s}}=\left\langle v \sigma_{n l}^{\mathrm{s}}\left(E_{\mathrm{i}}\right)\right\rangle=\int v \sigma_{n l}^{\mathrm{sl}}\left(E_{\mathrm{i}}\right) f\left(v_{\mathrm{i}}\right) \mathrm{d} v .
$$


The average is performed over Maxwellian velocity distribution function $f(v)$, where

$$
f(v)=\left(\frac{m^{0}}{2 \pi K T}\right)^{3 / 2} \exp \left(-\frac{m v^{2}}{2 \pi K T}\right),
$$

$T$ is the absolute temperature, $K$ is the Boltzmann constant and $v$ is the $\mathrm{CM}$ velocity. Since $\frac{3}{2} K T=\frac{1}{2} m v^{2}=E_{\mathrm{i}}$, we get from (24)

$$
\alpha_{n l}^{\mathrm{s}}=\int \frac{1}{m} \sigma_{n l}^{\mathrm{s}}\left(E_{\mathrm{i}}\right) f(v) \mathrm{d} E_{\mathrm{i}} .
$$

Total SRR rate is

$$
\alpha_{\mathrm{s}}=\sum_{n, l} \alpha_{n l}^{\mathrm{s}}
$$

Similarly, the total TSRR rate into $n l$-state through the intermediate $n^{\prime} l^{\prime}$ state is

$$
\alpha_{\mathrm{T}}=\sum_{n^{\prime}, l^{\prime}} \alpha_{n^{\prime} l^{\prime}}^{\mathrm{T}}
$$

where $\alpha_{n^{\prime} l^{\prime}}^{\mathbf{T}} \equiv\left\langle v_{\mathrm{i}} \sigma_{n^{\prime} l^{\prime}}^{\mathrm{T}}\left(E_{\mathrm{i}}\right)\right\rangle$.

\section{Results and discussions}

We have computed radiative recombination rates (Figs. 1a, b) and recombination cross sections (Fig. 2) of bare ions $\mathrm{Ne}^{10+}$ and $\mathrm{Ar}^{18+}$ with electrons at sub-zero temperatures, i.e., in the relative energy range $10^{-5} \mathrm{eV}$ to $10^{-3} \mathrm{eV}$.
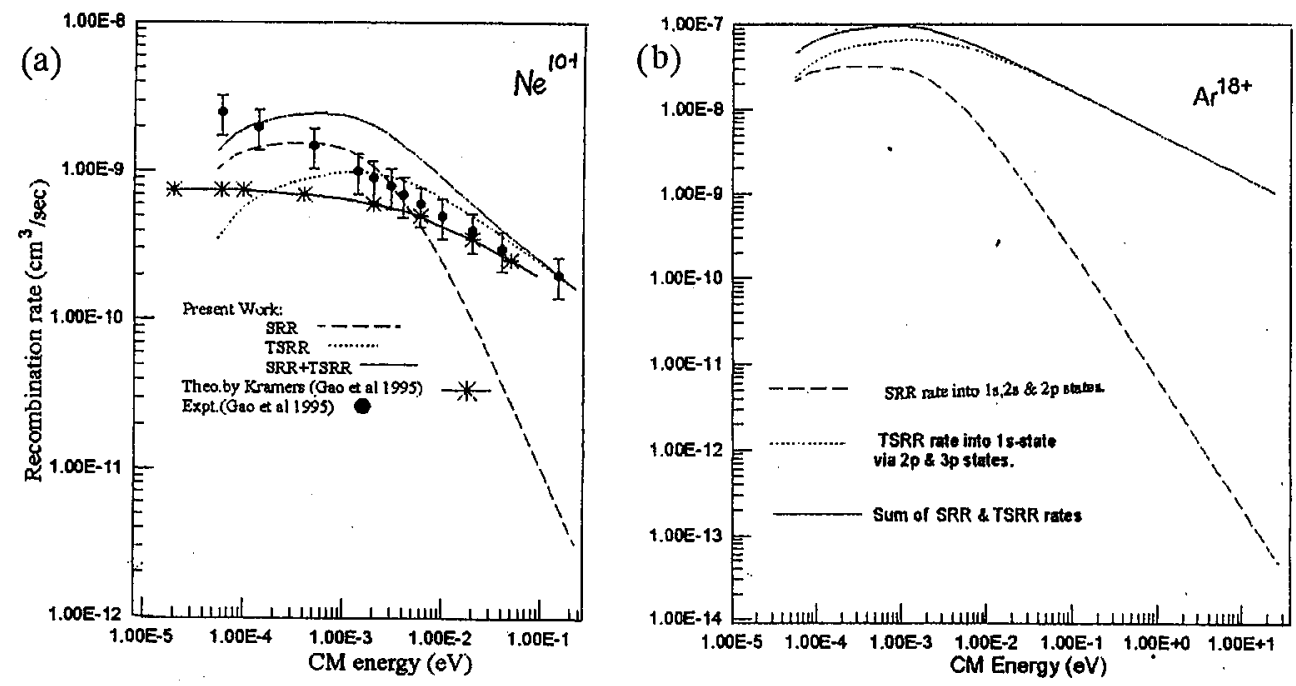

Fig. 1. Radiative recombination rate of $\mathrm{Ne}^{10+}$ (a) and $\mathrm{Ar}^{18+}$ (b) with electrons versus $\mathrm{CM}$ energy.

The spontaneous radiative recombinations for bare neon and argon ions are computed for the capture into $1 s, 2 s$ and $2 p$ states (Fig. 3). SRR cross section is found to vary inversely with incident energy $E_{\mathrm{i}}$. The dominant capture occurs in 


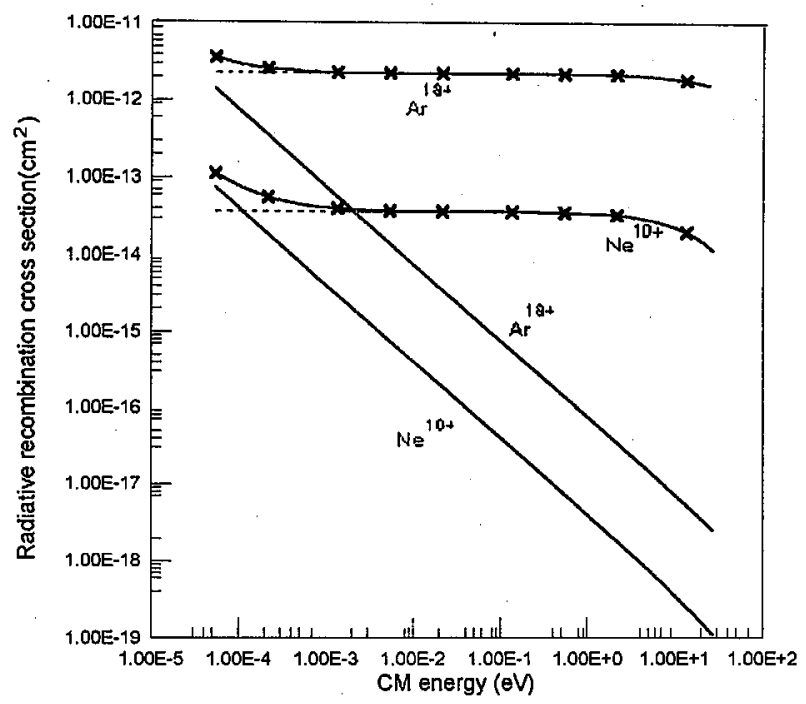

Fig. 2. Radiative recombination cross sections of $\mathrm{Ne}^{10+}$ and $\mathrm{Ar}^{18+}$ with electrons versus CM energy: - - a sum of SRR cross sections from $1 s, 2 s$ and $2 p$ states; $\cdots \cdots$ a sum of TSRR cross sections into $1 s$ state through $2 p$ and $3 p$ intermediate states; - $-\times-$ a sum of SRR and TSRR cross sections.

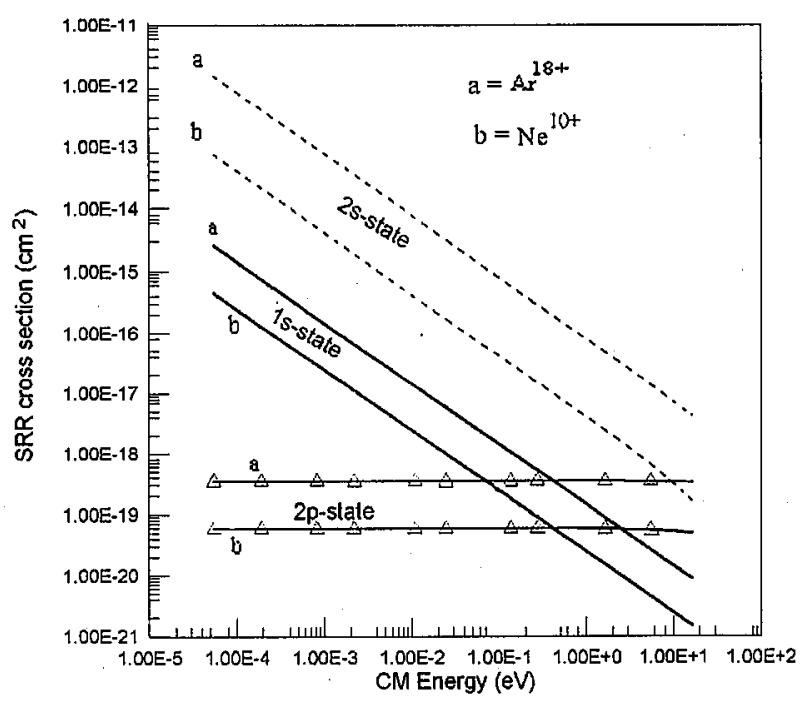

Fig. 3. SRR cross sections of $\mathrm{Ne}^{10+}$ and $\mathrm{Ar}^{18+}$ with electrons into $1 s, 2 s$ and $2 p$ states versus CM energy: $1 s$ state, $\cdots \cdots 2 s$ state, $\longrightarrow \triangle-2 p$ state.

$2 s$ state. The emitted photons in SRR have continuous spectra. The SRR cross section increases with $z$.

The two-step radiative recombination cross sections are computed for recombination into the ground state via $2 p$ and $3 p$ states. The TSRR through $3 p$ state 


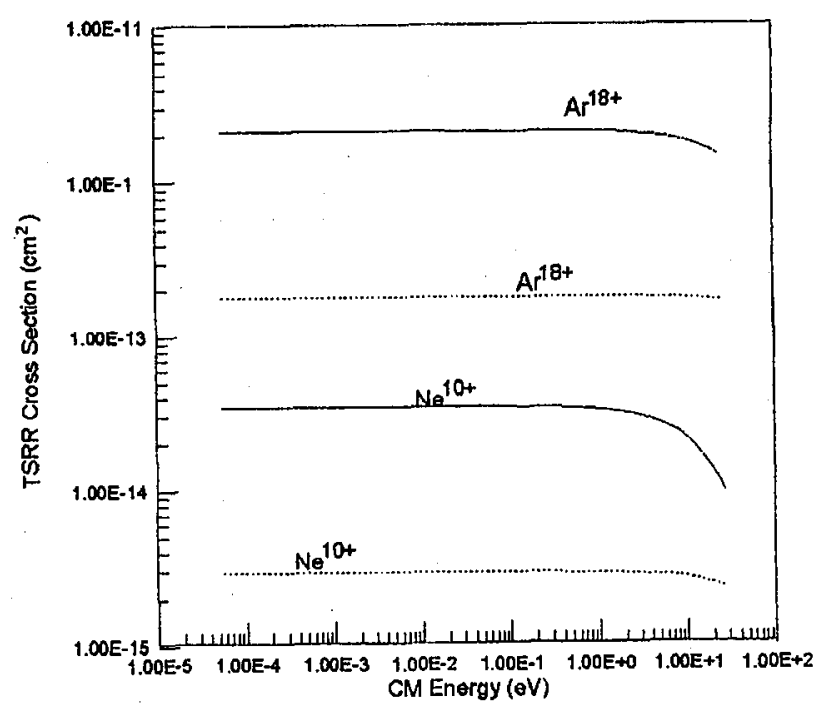

Fig. 4. TSRR cross sections of $\mathrm{Ne}^{10+}$ and $\mathrm{Ar}^{18+}$ with electrons into $1 s$ state versus $\mathrm{CM}$ energy, through the intermediate states: $2 p$ and $3 p$

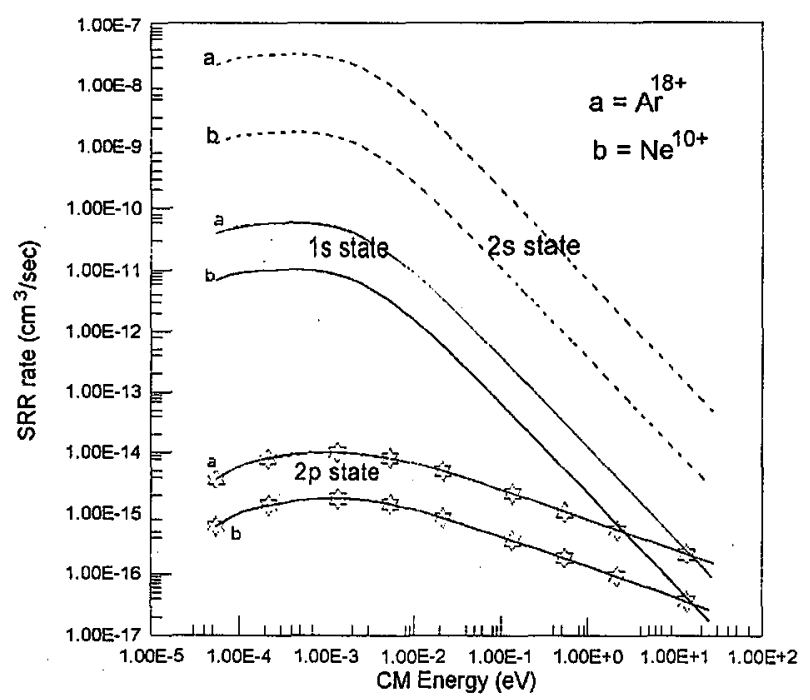

Fig. 5. SRR rates of $\mathrm{Ne}^{10+}$ and $\mathrm{Ar}^{18+}$ with electrons versus CM energy: $1 s$ state, $\ldots .2 s$ state, $-2 p$ state.

is greater than that through $2 p$ state. The TSRR cross sections are almost independent of $E_{\mathrm{i}}$ (Fig. 4) as long as $E_{\mathrm{i}}<1 \mathrm{eV}$. The emitted photons are naturally discrete. The cross section increases with $z$.

The recombination rates due to the SRR and TSRR channels considering the Maxwellian distribution of velocity are shown in Figs. 5 and 6, respectively. The present field theoretic results of sum of the SRR recombination rates of $\mathrm{Ne}^{10+}$ 


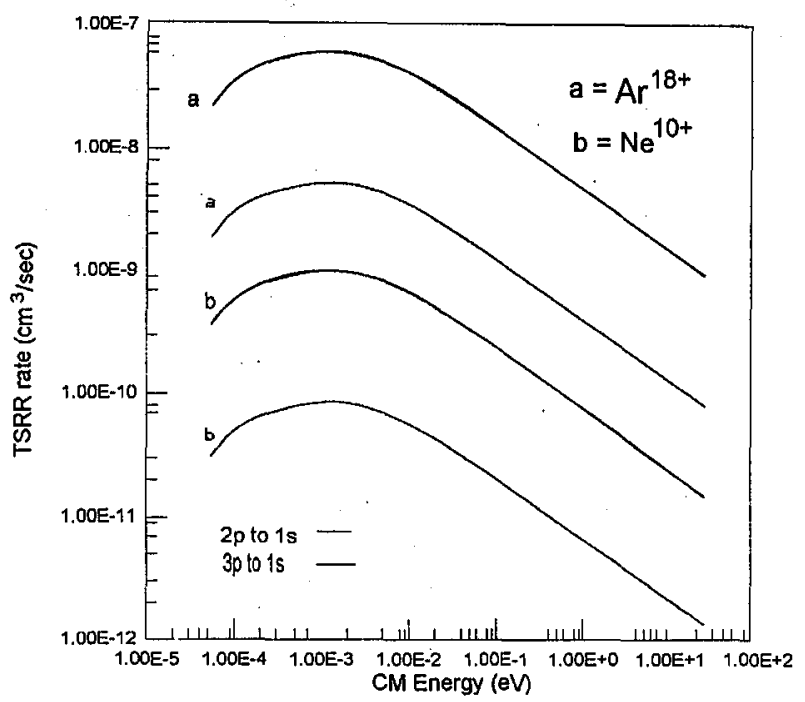

Fig. 6. TSRR rates of $\mathrm{Ne}^{10+}$ and $\mathrm{Ar}^{18+}$ with electrons versus $\mathrm{CM}$ energy.

and $\mathrm{Ar}^{18+}$ ions with sub meV electron are found to be greater than the sum of the TSRR rates (Fig. 1a, b). Above $1 \mathrm{meV}$ the sum of the rate coefficients from the TSRR channel follows the experimental fit curve for $\mathrm{Ne}^{10+}$, while the total SRR rates drop rapidly. The sum of the recombination rates from the SRR and TSRR channels near $1 \mathrm{meV}$, are found to be slightly higher than the experimental results (Fig. 1a).

\section{Conclusion}

The present paper is an attempt to show the contributions of the TSRR channel in the RR of bare ions with super cool electron. The presence of the SRR channel is well known in RR process. However, the experimental rate coefficients in the sub meV regions are several orders higher compared to the existing theoretical. results in the SRR channel. In the present paper, the field theory, which can explain most of the fundamental processes in nature, is applied to compute the SRR and the TSRR cross sections. Physically, the SRR and the TSRR are the two independent channels in RR process. Hence the question of interference between these channels does not arise. When the rate coefficients from these two chamnels are added together, the result is found to be comparable with the existing experimental da.ta. [8]. In actual experiment temperatures in the perpendicular and parallel directions of electron motion are different. In the present paper, for simplicity of calculation, we have taken temperature to be uniform in those directions while considering Maxwellian distribution of velocity for $R R$ rate calculations. Further, there is scope for improving the present result by including more intermediate states in the TSRR channel and more final states in the SRR channel. We hope to take up these in our future calculations. In conclusion, we like to suggest for experimental arrangements in RR process to identify the contributions from the SRR 
and TSRR channels by looking to their spectral signatures, namely, continuous and discrete characters of the spectral lines, respectively.

\section{Acknowledgment}

The authors thank UGC, N. Delhi, for the necessary support to complete the work.

\section{References}

[1] W.H. Tucker, Radiation Process in Astrophysics, MIT University Press, Cambridge (MA) 1975.

[2] M.J. Seaton, P.J. Storey, in: Atomic Process and Applications, Eds. P.G. Burke, B.L. Moisewitsch, North Holland, Amsterdam 1976, p. 133.

[3] A.H. Kramers, Philos. Mag. 46, 836 (1923).

[4] W. Gordon, Ann. Phys. (Leipzig) 2, 1013 (1929).

[5] M. Stobbe, Ann. Phys. (Leipzig) 7, 616 (1930).

[6] D.J. McLaughlin, Y. Hahn, Phys. Rev. A 43, 1313 (1991).

[7] S. Bhattacharyya, Acta Phys. Pol. A 86, 297 (1994).

[8] H. Gao, D.R. DeWitt, R. Schuch, W. Zong, S. Asp, M. Pajek, Phys. Rev. Lett. 75,4381 (1995).

[9] S. Bhattacharyya, K. Rinn, E. Salzborn, L. Chatterjee, J. Phys. B 21, 111 (1988).

[10] S. Bhattacharyya, A. Roy, S. Mitra, Fizika A 6, 149 (1997). 